\title{
Therapie des essentiellen Blepharospasmus mit Botulinumtoxin
}

\section{Therapy of Benign Essential Blepharospasm with Botulinum Toxin}

\author{
Autoren \\ Matthias Kaltenmaier ${ }^{1}$, Karoline Vanselow ${ }^{2}$, Jens Rollnik ${ }^{3}$, Matthias Maschke ${ }^{4}$
}

Institute

1 Universitätsklinik Heidelberg, Neurologie

2 St. Vincentius Kliniken Karlsruhe, Augenklinik

3 BDH-Klinik Hess. Oldendorf gGmbH, Institut für neurorehabilitative Forschung (InFo), Medizinische Hochschule Hannover

4 Krankenhaus der Barmherzigen Bruder Trier

Schlüsselwörter

Bleparospasmus, Therapie, Botulinumtoxin

Key words

blepharospasm, therapy, botulinum toxin

eingereicht 04.03.2018

akzeptiert 30.07.2018

Bibliografie

DOI https://doi.org/10.1055/a-0677-2122

ZUSAMMENFASSUNG

Der Blepharospasmus ist eine fokale Dystonie und eine seltene Erkrankung des Gehirns, bei der es zu unwillkürlichen Verkrampfungen der Lidschlussmuskulatur kommt. Die Diagnose wird klinisch gestellt und kann im Zweifel durch elektrophysiologische Untersuchungen bestätigt werden. Die Therapie der Wahl ist die lokale Injektion von Botulinumtoxin, die regelmäßig wiederholt werden muss.

\section{ABSTRACT}

Blepharospasm is a focal dystonia and a rare disease of the brain that causes involuntary spasms of the muscles responsible for eyelid closure. The diagnosis is made clinically and in unclear cases on the basis of results of electrophysiological tests. Therapy of choice consists of local injections with botulinum toxin that have to be repeated on a regular basis.

Fortschr Neurol Psychiatr 2019; 87: 355-360

(c) Georg Thieme Verlag KG Stuttgart · New York

ISSN 0720-4299

Korrespondenzadresse

Dr. Matthias Kaltenmaier

Universitätsklinik Heidelberg, Neurologie

INF 400

69120 Heidelberg

Matthias.Kaltenmaier@med.uni-heidelberg.de

\section{Einleitung}

Der Blepharospasmus (BSP) ist eine fokale Dystonie, bei der unwillkürliche tonische oder klonische Verkrampfungen hauptsächlich des $M$. orbicularis oculi auftreten [1]. Die Erkrankung tritt im mittleren bis höheren Lebensalter (Durchschnitt 56 Jahre) gehäuft bei Frauen (1,8:1) auf. Es handelt sich um eine seltene Störung mit einer Häufigkeit von 16-133 Betroffenen auf 1 Million [2]. In ca. $20 \%$ der Fälle ist der BSP mit oromandibulären und/oder fazialen Dystonien vergesellschaftet und wird dann als Meige-Syndrom bezeichnet. Die Symptome entwickeln sich über ca. 3
Jahre und bleiben dann in der Regel stabil, dabei kann sich die Störung auf benachbarte Muskelgruppen ausdehnen (z. B. cervikale Dystonie). Die Erkrankung kann bei schwerer Ausprägung bis zu einer funktionellen Blindheit führen, die Betroffenen sind durch den häufigen krampfartigen Lidschluss behindert und stigmatisiert, was zu beruflichen und sozialen Problemen führt.

In der vorliegenden Arbeit wird die Erkrankung an Hand der Anatomie, der Physiologie und Pathophysiologie, der Diagnostik und Differenzialdiagnostik näher beschrieben sowie die Therapie und Therapieprobleme beleuchtet. 
Wir möchten mit dieser Veröffentlichung allen Anwendern von Botulinumtoxin eine praxisnahe Hilfe zur Verfügung stellen und freuen uns über Rückmeldungen.

\section{Anatomie}

Beim BSP ist in erster Linie der M. orbicularis oculi betroffen. In etwa einem Viertel der Patienten können weitere Muskeln z. B. perinasal betroffen sein. Beim BSP vom Levatorinhibitionstyp führt der Versuch des Augenöffnens durch den M. levator palpebrae zu einer antagonistischen Augenschlussaktivität des palpebralen Anteils des M. orbicularis oculi.

Der Musculus orbicularis oculi besteht aus der Pars orbitalis und der Pars palpebralis. Zudem gibt es noch eine Pars lacrimalis, die dem Abfluss der Tränenflüssigkeit dient (siehe $>$ Abb. 1). Die Innervation erfolgt über die Rami temporales und zygomatici des N. facialis. Der Ursprung des Muskels ist der mediale Teil der Orbita, die Crista lacrimalis anterior und das Ligamentum palpebrae. Die Pars palpebralis setzt an der Haut der Oberlids und des Unterlids an, die Pars orbitalis dagegen weit gefächert an der Orbitahaut und der Stirn- sowie Wangenhaut. Die Pars lacrimalis setzt an der Tränendrüse an.

Funktionell führt die Kontraktion des M. orbicularis oculi zu einer Verengung der Lidspalte bis hin zum kompletten Lidschluss. Durch die Kontraktion wirkt er antagonistisch zum M. levator palpebrae und zu den sympathisch innervierten Mm. tarsalis superior und inferior.

Zu beachten ist bei der Injektion in den M. orbicularis oculi, dass die medialen Fasern der Pars orbitalis in den M. depressor supercilii (Augenbraue) einstrahlen.

Der M. levator palpebrae wird durch den Ramus superior des N. oculomotorius innerviert. Er entspringt der kaudalen Fläche der Ala minor des Os sphenoidale und kranial und ventral des Canalis opticus. Der Ansatz befindet sich am Knorpel und Haut des Oberlids. Die Funktion besteht in einer Hebung des Oberlids und stellt darüber die Weite der Lidspalte ein.

Der M. corrugator supercilii (Innervation Rami temporales des N. facialis) entspringt der Pars nasalis ossis temporalis und setzt an der Galea aponeurotica oberhalb des medialen Drittels der Augenbraue an. Er strahlt dabei in den M. orbicularis oculi ein. Funktionell zieht

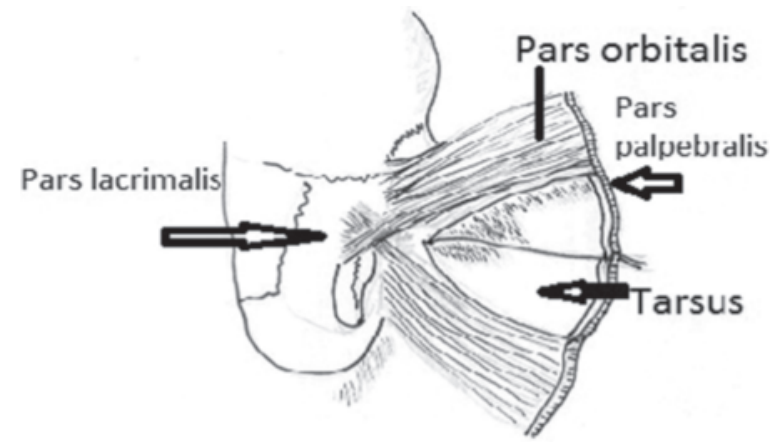

Abb. 1 Anatomie des M. orbicularis oculi mit seinen 3 Anteilen Pars orbitalis, Pars lacrimalis, Pars palpebralis. Ansicht von der Tarsusinnenseite auf den Muskel. der Muskel die Augenbrauen nach medial und bildet darüber die senkrechten Falten zwischen den Augenbrauen (sog. Zornesfalte).

Der M. procerus (Innervation Rami buccales des N. facialis) entspringt am unteren Anteil des Os nasale und setzt an der Stirnhaut zwischen den Augenbrauen an. Funktionell zieht er die medialen Augenbrauen in Richtung Nasenwurzel und verursacht darüber die tiefen Querfalten über der Nasenwurzel.

\section{Pathophysiologie}

Beim idiopathischen BSP handelt es sich um eine fokale Dystonie, die klinisch zu einer unwillkürlichen, bilateralen tonischen oder klonischen Kontraktion der periorbitalen Muskulatur führt [3]. Betroffen sind insbesondere der für den Lidschluss verantwortliche M. orbicularis oculi, aber auch der M. procerus, welcher Stirn und medialen Bereich der Augenbraue nach kaudal, und der M. corrugator supercilii, der die Augenbraue nach medial und unten zieht [3, 4]. Die Muskelkontraktionen greifen beim „reinen“ BSP nicht auf die übrige Gesichtsmuskulatur über, ansonsten wäre eher von der Diagnose einer fazialen bzw. oromandibulären Dystonie, z. B. dem Meige-Syndrom, auszugehen [5].

Hinsichtlich der Ätiologie werden ein primärer und ein sekundärer BSP differenziert. Während bei der primären dystonen, auch idiopathisch oder essenziell genannten Form keine augenfälligen Pathologien am Hirnparenchym nachweisbar sind, finden sich beim sekundären BSP neurologische (z. B. Alterationen im Thalamus, in den Basalganglien oder im Hirnstamm) oder ophthalmologische (z. B. Iritis, Konjunktivitis, Sicca-Symptomatik) Erkrankungen [3, 6].

Mit einer Verbesserung bildgebender Möglichkeiten gibt es allerdings auch beim primären BSP Hinweise auf makroskopische, volumetrische Veränderungen des Hirnparenchyms, z. B. eine bilaterale Zunahme der grauen Substanz im Putamen und eine Abnahme im Lobulus parietalis inferior [7]. Funktionell bildgebende Verfahren, insbesondere die funktionelle Magnetresonanztomografie (fMRT) legen eine Dyfunktion der sensorischen Verarbeitung bzw. der sensomotorischen Integration nahe [22].

Dass das Risiko für die Erkrankung an einem primären BSP in Familien mit Dystonie oder Tremor erhöht ist, spricht für genetische Ursachen [5]. Eine Assoziation von Polymorphismen des Torsin A- (DYT1) und D5 Dopamin-Rezeptor-Gens (DRD5) mit fokalen Dystonien ist bekannt, aber für den primären BSP spezifische genetische Veränderungen konnten nicht identifiziert werden [8]. Eine Untersuchung von Familien mit primärem BSP zeigte eine große phänotypische Variabilität der fokalen Dystonie, mit einem mutmaßlich autosomal-dominanten Erbgang und geringer Penetranz (ca. 20\%) [9].

\section{Diagnose und klinischer Befund}

Der BSP wird in erster Linie klinisch diagnostiziert. Über einen Zeitraum von 3-5 Jahren entwickelt sich das Vollbild der Erkrankung: (siehe - Abb. 2), danach tritt in der Regel keine weitere Verschlechterung mehr ein. Initial bemerken die Patienten einen häufigeren Lidschlag oder ein Fremdkörpergefühl, insbesondere verstärkt durch emotionale Belastung, grelles Licht oder Wind, Lesen, Autofahren und Gehen. Durch antagonistische Tricks kann der BSP kurzzeitig unterdrückt werden: Sprechen, Kauen, Lachen oder Singen, 
ähnlich der „geste antagoniste“ beim Torticollis spasticus ist dies individuell verschieden. Die Ausprägung kann sehr unterschiedlich sein. Zum Teil ist die Störung nur anamnestisch zu erheben und kaum bei der Untersuchung zu sehen, zum anderen kann sie bis zur funktionellen Blindheit führen. Sind weitere Muskeln des Gesichtes, des Mundes oder des Kiefers von den dystonen Bewegungen betroffen, spricht man vom Meige-Syndrom. Es werden verschieden Varianten des BSP beschrieben. Beim tonischen BSP kommt es zu einem persistierenden kräftigen unwillkürlichen Lidschluss, der auch passiv nicht überwunden werden kann. Überwiegend ist der orbitale Anteil des M. orbicularis oculi aktiv. Beim klonischen BSP kommt es zu unwillkürlichem Lidschluss, der weniger lang als bei der tonischen Form andauert und funktionell nicht so stark beeinträchtigt. Hierbei sind oft sowohl die pars orbitalis als auch die pars palpebralis des M. orbicularis oculi betroffen. Beim palpebralen BSP zeigt sich ein unwillkürlicher Lidschluss ohne das die pars orbitalis beteiligt ist. Es sind nur Muskelfasern des palpebralen M. orbicularis oculi aktiv, so dass hier auch von Lidöffnungsapraxie, Lidöffnungsinhibitionstyp oder Lidöffnungsakinese gesprochen wird. Hierbei handelt es sich aber um andere Erkrankungen, bzw. bei letzterem Begriff um eine irreführende und falsche Bezeichnung. Auffällig ist bei dieser Form, dass die Patienten durch Innervation des M. frontalis versuchen, die Augen zu öffnen. Auch kommt es bei raschem willkürlichem Augenöffnen zu einer kurzen Verzögerung der Lidöffnung, während der orbitale Anteil des orbicularis oculi bereits erschlafft ist.

Die Inhibitionsstörung der Lidöffnung ist vom BSP abzugrenzen und wird daher bei Differentialdiagnose besprochen.

Ein einseitiger BSP tritt in $5 \%$ der Fälle auf. Dieser geht aber häufig im weiteren Verlauf in die beidseitige Form über. Hierbei ist zu beachten, dass sich die gleichseitige Augenbraue bei der Kontraktion des M. orbicularis oculi senkt, was als „Charcot-Zeichen“ beschrieben wird, im Gegensatz zum Spasmus hemifacialis, bei dem die Augenbraue angehoben wird (siehe Differentialdiagnose).

\section{Diagnostik und Differentialdiagnosen}

Obwohl die klinische Diagnose im Vordergrund steht, empfehlen einige Autoren zusätzliche Untersuchungen zum Ausschluss verschiedener Differentialdiagnosen [10]. In jedem Falle sollte eine augenärztliche Untersuchung erfolgen, um z.B. Reizzustände am Auge auszuschließen. Ein MRT des Schädels zum Ausschluss einer Basalganglienerkrankung gerade beim jüngeren Patienten (z. B. Morbus Wilson) ist sinnvoll, auch kann ein Gefäß-Nerven-Konflikt bei einseitigem Auftreten und differentialdiagnostisch zu erwägendem Spasmus hemifacialis nachgewiesen werden. Zur Abgrenzung zum Spasmus hemifacialis hilft auch das Babinski-Zeichen-2, da beim Spasmus hemifacialis bei der Kontraktion des M. orbicularis oculi die Augenbraue gleichzeitig und unwillkürlich durch Aktivierung des $M$. frontalis angehoben wird. Hier kann auch der Blinkreflex mit Nachweis einer lateral spread response [11] hilfreich sein. Der Blinkreflex kann auch zur Differentialdiagnostik bei möglichen Synkinesien nach peripherer Facialisparese genutzt werden. Hierbei tritt beim spontanen oder willkürlichen Lidschlag synchron eine Kontraktion weiterer vom N. facialis innervierter Muskeln auf bzw. bei Mundbewegungen kommt es zum Augenschluss (siehe auch bei [12]).

Die Inhibitionsstörung der Mm. levator palpebrae und orbiccularis oculi ist klinisch vom BSP nicht zu unterscheiden. Es kommt hierbei zum Ausfall der gegenseitigen Inhibition der antagonistisch wirkenden Muskeln. Die Erkrankung ist selten und wird mit diffusen Gehirnerkrankungen in Zusammenhang gebracht. Im EMG zeigt sich eine synchrone Aktivität in beiden Muskeln beim Versuch, die Augen zu öffnen. Ein Therapieversuch mit Botulinumtoxin ist sinnvoll [19].

Die Lidheberapraxie als Differenzialdiagnose ist klinisch nicht einfach von einem palpebralen BSP zu unterscheiden. Bei dieser Apraxie gelingt es aber sowohl dem Patienten als auch dem Untersucher leicht, die Augen passiv zu öffnen, da ja keine unwillkürliche Kontraktion vorliegt. Die Augen bleiben auch bis zum nächsten Lidschlag offen. Im EMG zeigt sich keine Aktivität im M. levator palpebrae und im M. orbicularis oculi pars palpebralis. Die Ursachen der Lidheberapraxie sind vaskuläre oder entzündliche Gehirnerkrankungen oder neurodegenerative Gehirnerkrankungen wie z. B. MSA, PSP, CBD. Die Behandlung mit Botulinumtoxin ist in diesem Falle eher nicht wirksam.

Als weitere Differentialdiagnosen kommen noch die okuläre Myasthenie und faziale Myokymien in frage.

\section{Therapie}

Therapie der ersten Wahl des BSP ist die Injektion von Botulinumtoxin. Für folgende Medikamente besteht eine Zulassung: Onabotulinumtoxin-A (Botox ${ }^{\circledR}$ ), Abobotulinumtoxin-A (Dysport ${ }^{\circledR}$ ) und Incobotulinumtoxin-A $\left(X_{\text {Xeomin }}{ }^{\circledR}\right)$. Es existieren verschiedene Injektionsschemata, wobei alle Injektionen subcutan ausgeführt werden, da die Gesichtsmuskeln keine Faszie besitzen und somit nicht zwingend intramuskulär injiziert werden muss. In den Fachinformationen der Hersteller wird nur bei Dysport [17, 20, 21] die Lokalisation der Injektionsstellen exakt beschrieben und dargestellt (60 bis 120 Einheiten [E] je Auge an vier bis sechs Injektionsstellen), bei den beiden anderen Toxinen wird nur eine Gesamtmenge angegeben (25 bis $100 \mathrm{E}$ je Auge). Bei der tonischen und klonischen Form wird ein 3oder 4-Punkt-Schema empfohlen. Die Gebrauchsinformation von Dysport gibt als Lokalisation der Injektion die Verbindung zwischen dem präseptalen (palpebralen) und orbitalen Teil des M. orbicularis oculi an. Ein Autor empfiehlt die Injektion am knöchernen Orbitarand [13], andere oberhalb des oberen Orbitarandes bzw. periorbital ohne nähere Angaben [14, 15]. Diese Injektionspunkte werden auch als lidrandfern beschrieben. Pro Injektion werden bei Dysport $10 \mathrm{E}$ (bis 20E) medial und $20 \mathrm{E}$ (bis 40E) lateral angegeben, bei Botox und Xeomin 2-6 E an jeder Injektionsstelle. Ein Autor unterscheidet bei Dysport und Botox/Xeomin die Lokalisationen der 


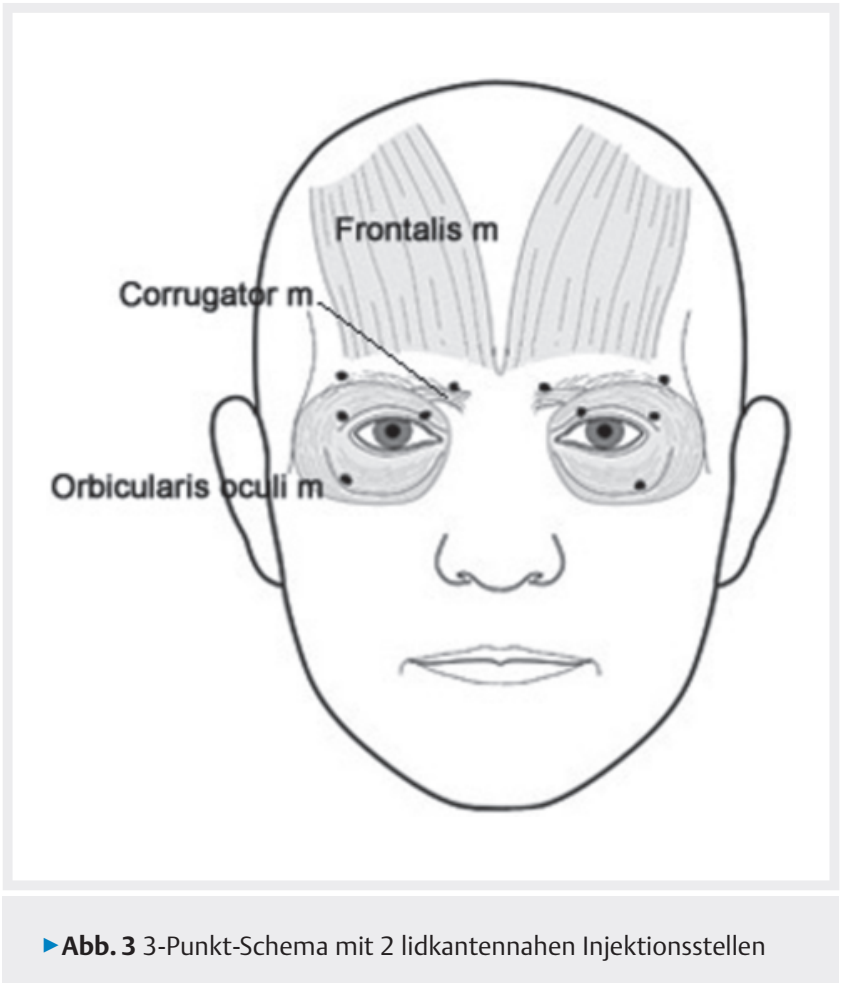

Injektionsstellen [14]. Bei der palpebralen Form oder bei ausgeprägtem BSP mit starker funktioneller Beeinträchtigung werden zusätzlich ein bis zwei palpebrale (lidkantennahe) Injektionen mit 5-10 E Dysport bzw. 1-2 E Botox/Xeomin empfohlen. Zur Vereinfachung und Standardisierung der Behandlung empfehlen die Autoren zunächst ein 3-Punkt-Schema mit Injektionsstellen am knöchernen Orbitarand medial und lateral oben sowie lateral unten mit jeweils 10 E Dysport oder 4 E Botox / Xeomin. Die Stichrichtung der oberen Injektionen sollte vom Lid weg erfolgen (siehe $>$ Abb. 3), um eine Ptose durch Diffusion des Toxins zum M. levator palpebrae möglichst zu vermeiden.

Bei schlechtem Ansprechen sollte dann ein 4-Punktschema mit höherer Dosis z. B. 4x15 E Dysport bzw. 4x6 E Botox/Xeomin eingesetzt werden. Zusätzlich sollten dann auch 2 Injektionen lidkantennah mit je 5 E Dysport bzw. 1 Einheit Botox/ Xeomin ergänzt werden, um eine Beteiligung des palpebralen Anteils des M. orbicularis oculi nicht zu übersehen. In Sonderfällen beim reinen palpebralen Typ kann eine lidkantennahe Injektion mit geringen Mengen ausreichend sein. Je nach Wirkung kann die Dosis noch bis 120 E Gesamtdosis Dysport oder $100 \mathrm{E}$ Botox/Xeomin je Auge laut Fachinformation erhöht werden. Diese Mengen werden aber erfahrungsgemäß kaum notwendig sein. Bei einer Lidöffnungsapraxie kann ein Therapieversuch mit lidkantennaher Injektion von 5-20 E Dysport bzw. 1-5 E Botox/ Xeomin erfolgen [14], allerdings kann auch eine Verschlechterung eintreten [16].

Die Wiederholung der Injektionen erfolgt in der Regel alle 3 Monate. Je nach Wirkdauer kann das Intervall verkürzt oder verlängert werden, wobei Intervalle kürzer als 8 Wochen zur Vermeidung von Antikörperbildung nicht unterschritten werden sollten. Zubereitung und Verwendung des Botulinumtoxins:

Die Präparate Botox ${ }^{\circledR}$, Xeomin ${ }^{\circledR}$ und Dysport ${ }^{\circledR}$ liegen in Pulverform vor - sie dürfen nur mit steriler Natriumchloridinjektionslösung
$9 \mathrm{mg} / \mathrm{ml}(0.9 \%)$ rekonstituiert werden. Die Verwendung von aqua ad iniectabilia führt zu massivem Brennen und Schmerzen nach der Injektion.

Xeomin $^{\circledR}$ und Dysport ${ }^{\circledR}$ : Chemische und physikalische Stabilität der gebrauchsfertigen Lösung wurde für $24 \mathrm{~h}$ bei $2^{\circ}-8^{\circ} \mathrm{C}$ nachgewiesen.

Botox ${ }^{\circledR}$ : Studien zur Wirksamkeit haben gezeigt, dass das Arzneimittel nach Rekonstitution bis zu 5 Tage bei $2^{\circ}-8^{\circ} \mathrm{C}$ gelagert werden darf.

Jedoch gilt für alle: Sofern die Herstellung der gebrauchsfertigen Zubereitung nicht unter kontrollierten und validierten aseptischen Bedingungen erfolgt, ist diese nicht länger als $24 \mathrm{~h}$ bei $2^{\circ}$ bis $8^{\circ} \mathrm{C}$ aufzubewahren und zu verwenden. $[17,20,21]$

\section{Fallstricke und Therapieprobleme in der Botulinumtoxin-Therapie des BSP}

Bei der Injektion mit Botulinumtoxin sind anatomische Strukturen zu beachten, die durch die folgende Abbildung verdeutlicht werden sollen (siehe $>$ Abb. 4).

Je nach Wahl der Injektionspunkte, wie sie unter Diagnostik beschrieben wurden, sind folgende Punkte zu beachten:

Sicca-Symptomatik: Das Sekret der Glandula lacrimalis wird über 6-12 Ausführungsgänge in das Gewölbe des Fornix conjunctivae geleitet und mit dem Lidschlag über dem Bulbus und der Hornhaut verteilt. Eine Diffusion des Toxins in den Bereich der Glandula kann zur Verstärkung einer Sicca-Symptomatik führen. Die Beeinflussung des Lidschlags und die Verminderung der Lidschlusskraft können zu einer Störung des Tränentransports und der Tränenverteilung führen. Weiter außerhalb liegende Injektionen können zu Sekretionsstörungen entfernterer Drüsen führen.

Lagophthalmus: Durch dosisabhängige Verminderung der Lidschlusskraft kann es zu einem Lagophthalmus am Tag, evtl. auch nur in der Nacht kommen. Das Auge steht offen, es fehlt die Benetzung von Hornhaut und Bindehaut mit der Gefahr der Expositionskeratitis und Ulceration. Dies betrifft insbesondere die Zeit der stärksten muskelschwächenden Wirkung nach 2-3 Wochen. Sollte ein Lagophthalmus auftreten oder aber zur Linderung des BSP in Kauf genommen werden, sollte man Injektionen direkt über der Glandula lacrimalis vermeiden und vor der Injektion ggf. eine Konjunktivitis sicca (Schirmer-Test I und II) nachweisen. Tränenersatzmittel tagsüber und Gel- oder Salbenapplikation zur Nacht müssen gegebenenfalls eingesetzt werden.

Epiphora: Dosisabhängig bzw. nach Injektion in Unterlidmitte kann es zur Verminderung der Lidschlusskraft bzw. einem Ektropium mit Epiphora kommen. Eine Injektion im medialen Anteil des Unterlids kann zur Schwächung der Pars lacrimalis (Horner-Muskel) des M. orbicularis oculi führen ( $\triangleright$ Abb. 1 ), die die Canaliculi und den Saccus lacrimalis umschließen und das Ansaugen und Abpumpen der Tränen Richtung Nase kontrollieren mit der Folge einer Tränenabflußstörung. Bei regelmäßigem Auftreten dieser unerwünschten Wirkung dürfen diese Punkte nicht mehr injiziert werden.

Hämatom und Ekchymosen: Eine Hämatombildung ist möglich durch Injektion im Bereich der verzweigten und miteinander 


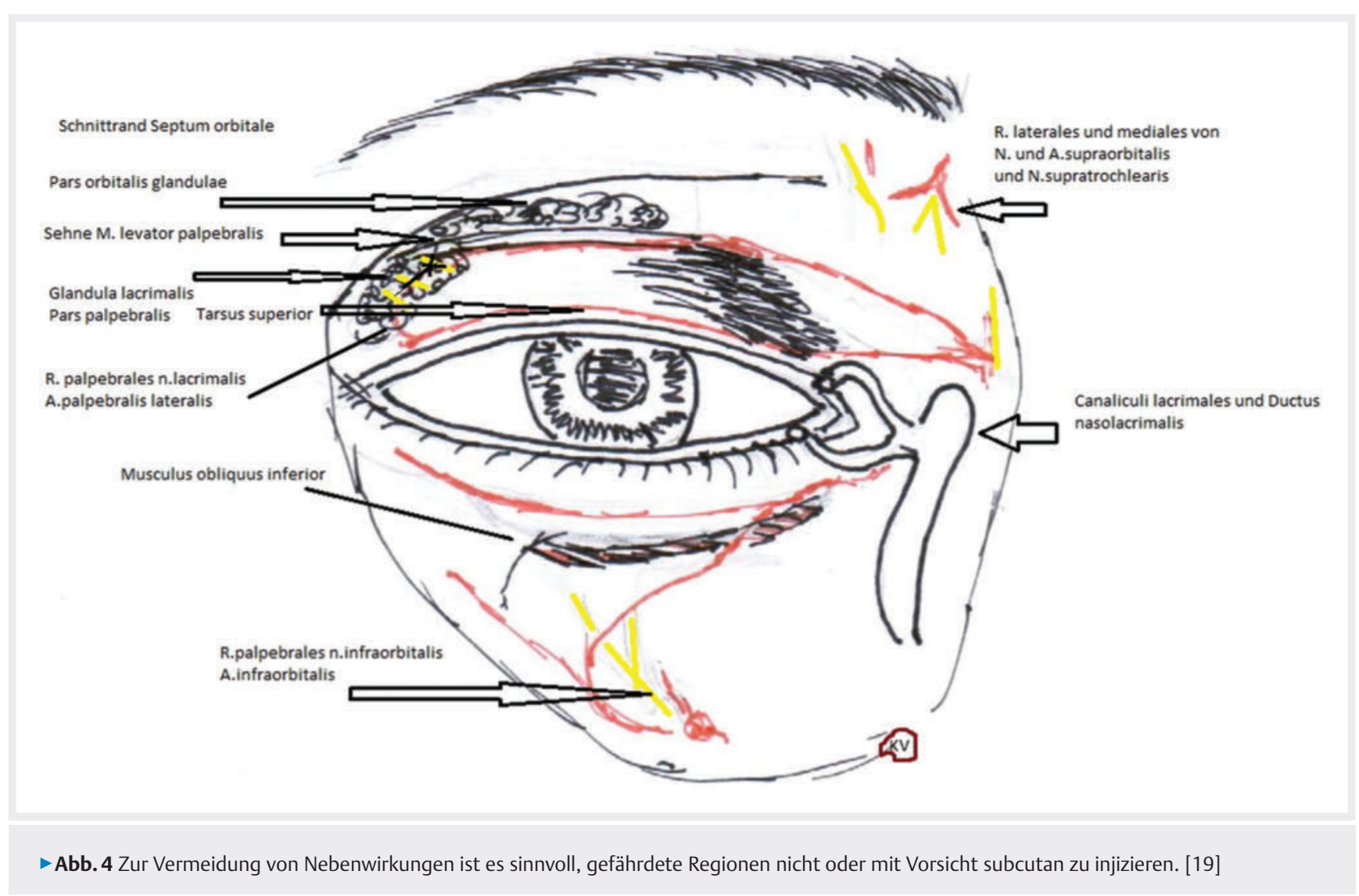

vernetzten Äste der Arteria palpebralis des Ober- und Unterlides und tritt nicht selten auf. Sie kann durch Kompression gut kontrolliert werden, so dass eine Therapie mit Antikoagulantien keine Kontraindikation darstellt.

Unerwünschte Wirkung auf benachbarte Muskeln:

- Ptosis:

Eine Injektion im Bereich des medialen Oberlids kann zur Diffusion in den M. levator palpebrae und damit zur temporären Ptosis führen. Auf Kanülenführung von der Mitte in Richtung zum inneren oder äußeren Augenwinkel sollte daher geachtet werden.

- Diplopie:

Die geraden Augenmuskeln sind für eine Lähmung nicht so anfällig, da sie ca. $6 \mathrm{~mm}$ hinter dem Limbus am Bulbus ansetzen. Im medial oberen Orbitabogen befindet sich die Trochlea, durch die die Sehne des M. obliquus superior zieht, um dann fächerförmig auf dem Bulbus anzusetzen.

Der M. obliquus inferior hat seinen Ursprung am Os lacrimale im unteren medialen Bogen der Orbita (Siehe $>$ Abb. 4). Er verläuft unter dem $M$. rectus inferior nach außen. Durch Toxin-Diffusion kann es zu vertikaler Diplopie und Excyclorotation kommen, die z.B. zur passageren Fahruntauglichkeit führen kann. Hierbei kann die Anpassung einer Prismenfolie bzw. eine temporäre Vollokklusion eines Auges notwendig werden. [18]

Mangelndes Ansprechen der Botulinumtoxin Therapie: Bei fehlendem oder schlechtem Ansprechen der Botulinumtoxintherapie wird die Dosis erhöht, und zwar pro Punkt von anfangs 4 E Botox / Xeomin auf 6-10 bzw. von 10 E Dysport auf 20-40 E. Auch eine
Umstellung auf ein anderes Botulinumtoxin-A Präparat kann zu einem besseren Ansprechen führen [4]

Variation der Punktewahl bei Unterformen des essentiellen BSP: Bei der rein palpebralen Form bzw. einer Mitbeteiligung der Pars palpebralis des $M$. orbicularis oculi erfolgen alleine oder zusätzlich 1 oder 2 lidrandnahe Injektionen in die Pars palpebralis. Diese führen zu einer höheren Responderrate und einer längeren Dauer der maximalen Wirkung (siehe auch Therapie).

Injiziert man bei der palpebralen Form das Botulinumtoxin nur in die orbitalen Fasern, kann es zu einer Verstärkung des unwillkürlichen Augenschlusses kommen. Injektionen in die Antagonisten des M. orbicularis, z. B. den Venter frontalis des M. occipitofrontalis, müssen vermieden werden. Eine Injektion in den M. depressor supercilii ist dagegen gestattet.

Bei einer Störung der Inhibition der Lidöffnung des M. levator palpebrae erbringt die lidrandnahe Injektion der Pars palpebralis meist keine Symptomfreiheit, eine Besserung kann dennoch eintreten, da dieser geschwächte Muskel dann vom M. levator palpebrae überwunden werden kann.

Bei der Therapie der bereits besprochenen Differentialdiagnose Lidapraxie ist ein Therapieversuch mit Botulinumtoxin statthaft, bei Versagen kommt dann eine Lidsuspension mit Frontalisschlingen-Operation in Frage.

Ein sekundäres Therapieversagen durch neutralisierende Antikörper ist bei wiederholtem Nichtansprechen zu bedenken. Dies ist jedoch beim essentiellen BSP wegen der geringen Toxinmenge sehr selten. [19] 


\section{FAZIT FÜR DIE PRAXIS}

Mit den verschiedenen Botulinumtoxinen steht eine wirkungsvolle und sichere Therapie zur Behandlung des Blepharospasmus zur Verfügung. Diese kann prinzipiell unbegrenzt angewendet werden und führt bei Beachtung der genannten Punkte in einem sehr hohen Prozentsatz der Betroffenen zu einer guten bis sehr guten Besserung der Symptome.

\section{Interessenkonflikt}

Matthias Kaltenmaier erhielt Unterstützung bei Fortbildungen und Honorare für Vorträge der Firmen Pharm Allergan, Ipsen Pharma und Merz Pharma.

Matthias Maschke erhielt Honorare für Vorträge und Workshops sowie Unterstützung von Fortbildungsveranstaltungen und Studien von Pharm Allergan, Ipsen Pharma und Merz Pharma.

Die anderen Autoren haben keinen Interessenkonflikt.

\section{Literatur}

[1] Marsden CD. The problem of adult-onset idiopathic torsion dystonia and other isolated dyskinesias in adult life (including blepharospasm, oromandibular dystonia, dystonic writer's cramp, and torticollis, or axial dystonia). Adv Neurol 1976; 14: 259-76.

[2] Defazio G, Abbruzzese G, Livrea P et. al. Epidemiology of primary dystonia. Lancet Neurol 2004; 3: $673-8$.

[3] Ceballos-Baumann AO. Dystonien. In: Conrad B, Ceballos-Baumann AO (Hrsg.): Bewegungsstörungen in der Neurologie. Thieme-Verlag, Stuttgart 1996: 89-140.

[4] Mattle H, Mumenthaler M. Neurologie. Thieme-Verlag, Stuttgart 2013: 209.

[5] Lücking $\mathrm{CH}$, Amtage F, Hummel S, Hornyak M, Zucker B, Hellwig B. Basalganglienerkrankungen. In: Hufschmidt A, Lücking $\mathrm{CH}$, Rauer S (Hrsg.) Neurologie compact. Stuttgart, Thieme; 2013: 337-386.

[6] Hallett M, Evinger C, Jankovic J et. al. Update on blepharospasm. Neurology 2008; 71: 1275-1282.
[7] Etgen T, Muhlau M, Gaser C et. al. Bilateral greymatter increase in the putamen in primary blepharospasm. I Neurol Neurosurg Psychiatry 2006; 77: $1017-1020$.

[8] Clarimon J, Brancati F, Peckham E et al. Assessing the role of DRD5 and DYT1 in two different case-control series with primary blepharospasm. Mov Disord 2007; 22: 162-166.

[9] Defazio G, Martino D, Aniello MS et. al. A family study on primary blepharospasm. J Neurol Neurosurg Psychiatr 2006; 77: 252-254.

[10] Reichel G; Blepharospasmus. In Reichel G, Therapieleitfaden SpastikDystonie. Bremen: Uni-Med; 2012: 130-132

[11] Glocker FX, Lücking $\mathrm{CH}$. Elektrische und magnetische Reiztechniken zur Diagnostik der Fazialisparese und des Hemispasmus facialis. Klin Neurophysiol 1998; 29(2): 59-65

[12] Jost WH, Laskawi R, Palmowski-Wolfe A et al. Therapie des Spasmus hemifacialis mit Botulinumtoxin. Fortschr Neurol Psychiatr 2017; 85: $1-5$

[13] Asmus F; Tübinger Grundkurs. Michelstadt: Verlag für Didaktik in de Medizin; 2006

[14] Naumann, M. Botulinumtoxin. 1. Aufl. Bremen: Uni-Med; 1998

[15] Reichel G. Injektionsorte und Dosen für Btx. In: Reichel G. Therapieleitfaden Spastik-Dystonie. Bremen: Uni-Med; 2012: 137-138

[16] Reichel G; Lidheberapraxie. In Reichel G. Therapieleitfaden SpastikDystonie. Bremen: Uni-Med; 2012:139-140

[17] Ipsen Pharma. Fachinformation Dysport ${ }^{\circledR} 300$ Einheiten/ 500 Einheiten. Stand Dezember 2016. Im Internet: ipsen-pharme.de; Stand 03.12.2017

[18] Roggenkämper P. Was tun bei unzureichender Wirkung von BotulinumToxin in der Behandlung von Lidkrämpfen? Der Ophthalmologe 2007; 104: 763-766

[19] Reichel G, Stenner A, Hermann W (2009): Palpebrale Variante des BSPAbgrenzung zur Lidöffnungsapraxie und zur Inhibitionsstörung durch synchrone EMG-Ableitungen Akt Neurolog 2009; 36: 60-64

[20] Allergan. Fachinformation Botox 50/ $100 / 200$ Allergan-Enheiten. Stand Januar 2017. Im Internet: www.allergan.de; Stand 03.12.2017

[21] Merz. Fachinformation Xeomin 50/100/200. Stand September 2017. Im Internet: www.gelbe-liste.de; Stand 03.12.2017

[22] Huang XF, Zhu MR, Shan P, Pei CH, Liang ZH, Zhou HL, Ni MF, Miao YW, Xu GQ, Zhang BW, Luo YY. Multiple Neural Networks Malfunction in Primary Blepharospasm: An Independent Components Analysis. Front Hum Neurosci 2017; 11: 235. 